\title{
Achieving superior two-way actuation by the stress-coupling of nanoribbons and nanocrystalline shape memory alloy
}

Shijie Hao, ${ }^{\dagger} \dagger$ Yinong Liu, ${ }^{\ddagger}$ Yang Ren, ${ }^{\S}$ Daqiang Jiang, ${ }^{\dagger}$ Feng Yang, $^{\dagger}$ Daoyong Cong, ${ }^{\perp}$ Yandong Wang, ${ }^{\perp}$ and Lishan Cui ${ }^{\dagger}$

${ }^{\dagger}$ State Key Laboratory of Heavy Oil Processing, China University of Petroleum, Beijing 102249, China

${ }^{\ddagger}$ School of Mechanical and Chemical Engineering, The University of Western Australia, Crawley, WA 6009, Australia

${ }^{\S}$ X-ray Science Division, Argonne National Laboratory, Argonne, Illinois 60439, USA

${ }^{\perp}$ State key laboratory for Advanced Metals and Materials, University of Science and Technology, Beijing 10083, China

\begin{abstract}
Inspired by the driving principle of traditional bias-type two-way actuators, we developed a novel two-way actuation nanocomposite wire in which a massive number of $\mathrm{Nb}$ nanoribbons with ultra-large elastic strains are loaded inside a shape memory alloy (SMA) matrix to form a continuous array of nano bias actuation pairs for two-way actuation. The composite exhibits a two-way actuation strain of 3.2\% during a thermal cycle and an actuation stress of $934 \mathrm{MPa}$ upon heating, which is about twice higher than that ( $500 \mathrm{MPa}$ ) found in reported two-way SMAs. Upon cooling, the composite shows an actuation stress of $134 \mathrm{MPa}$ and a mechanical work output of $1.08 * 10^{6} \mathrm{~J} / \mathrm{m}^{3}$, which are about three and five times higher than that of reported two-way SMAs, respectively. It is revealed that the massive number of $\mathrm{Nb}$ nanoribbons in compressive state provides the high actuation stress and high work output upon cooling and the SMA matrix with high yield strength offers the high actuation stress upon heating. Compared to traditional bias-type two-way actuators, the two-way actuation composite with small volume and simple construct is in favour of the miniaturization and simplification of actuators.
\end{abstract}

KEYWORDS: Two-way actuation, Coupling stress, Nanoribbons, Shape memory alloy, Nanocomposite

\section{INTRODUCTION}

High-performance actuator materials capable of outputting high actuation stresses and large actuation strains are of high demand for many applications, such as micro-robots, ${ }^{1-3}$ micro air vehicles, ${ }^{3-4}$ artificial organs ${ }^{5-6}$ and prosthetic devices. ${ }^{7}$ Many candidate materials have been studied, such as piezoelectric ceramics, ${ }^{8}$ magnetostrictive alloys, ${ }^{9}$ shape memory alloys (SMAs) ${ }^{10-11}$ and electrostatic polymers. ${ }^{12}$ Among them, SMAs have the highest mechanical 
work output capacity $\left(\sim 10^{7} \mathrm{~J} / \mathrm{m}^{3}\right),{ }^{13-14}$ typically 100 times of that of piezoelectric ceramics and 50 times of electrostatic polymers. ${ }^{13-14}$

SMAs typically exhibit the ability to output large actuation strains of $5 \%$ and actuation stresses up to $500 \mathrm{MPa}$, producing a mechanical work output of $2.5^{*} 10^{7} \mathrm{~J} / \mathrm{m}^{3}$. ${ }^{13-14}$ However, such properties are associated with the reverse transformation from deformed martensite to austenite, thus are only achievable upon heating (effectively by converting the thermal energy into mechanical energy). ${ }^{13}$ Upon cooling, SMAs are usually passive and may exhibit small strain output or very low actuation stress (5 50 MPa), ${ }^{13,15-16}$ thus very low work output. This severely restricts their applications as thermostat two-way actuators.

To overcome this shortfall, a bias spring system is often required to work with the SMA actuator in order to achieve two-way actuation. ${ }^{10,16-17}$ The bias spring system provides the actuation upon cooling by overcoming the weaker SMA component and upon heating is overcome by the stronger SMA. This drastically complicates the structure design and the control system severely diminishes their advantages going against in the miniaturization and simplification of actuator designs.

Inspired by the driving principle of bias-type two-way actuators, we envisaged a novel two-way actuation nanocomposite concept in which a massive number of nano-springs are loaded inside a SMA matrix to form a continuous array of nano bias actuation pairs for reversible two-way actuation. The nano-springs are simple metal nanowires, which have been shown to be able to exhibit up to $6 \%$ pure elastic strains in perfect compatibility with SMAs. ${ }^{18}$ Such composite of massive number of nano bias pairs is expected to be able to provide high actuation stress and high work output upon cooling, whist still maintains its small volume and simple construct, which are essential for the miniaturization and simplification of actuators.

To verify this design concept, we fabricated an in-situ Nb nanoribbon-nanocrystalline NiTi SMA composite wire (Figure S1) by severe cold wire-drawing of a Nb-NiTi hypo-pseudoeutectic casting and annealing treatment. By prior thermo-mechanical treatments including a $12 \%$ tensile deformation at room temperature and a $20 \rightarrow 200 \rightarrow 20{ }^{\circ} \mathrm{C}$ heating-cooling cycle, a high coupling stress between the superelastic $\mathrm{Nb}$ nanoribbons and the shape memory nanocrystalline NiTi matrix was induced (Figure S2), to realize the design of concept of massive number of nano-bias active systems in the composite wire.

\section{EXPERIMENT SECTION}

An ingot of $25 \mathrm{~kg}$ in weight with a composition of $\mathrm{Ni}_{45} \mathrm{Ti}_{45} \mathrm{Nb}_{10}$ (at.\%) was prepared by vacuum induction melting. The ingot was hot-forged at $850{ }^{\circ} \mathrm{C}$ into a rod of $16 \mathrm{~mm}$ in diameter and hot-drawn at $750{ }^{\circ} \mathrm{C}$ into a thick wire of $2 \mathrm{~mm}$ in diameter. The hot-drawn wire was cold-drawn into a thin wire of $0.5 \mathrm{~mm}$ in diameter at room temperature with the intermediate annealing at $700{ }^{\circ} \mathrm{C}$. The samples were cut from the cold-drawn wire and annealed at $450{ }^{\circ} \mathrm{C}$ for $10 \mathrm{~min}$ followed by air cooling. The $12 \%$ tensile deformation was performed using an Instron testing machine at a strain rate of $1 \times 10^{-4} \mathrm{~s}^{-1}$ at room temperature. The heating-cooling cycle $\left(20^{\circ} \mathrm{C} \rightarrow 200{ }^{\circ} \mathrm{C} \rightarrow 20^{\circ} \mathrm{C}\right)$ was performed using an ordinary air heating furnace.

Microstructure was analyzed using a TEM (FEI Tecnai G2 F20) operated at a voltage of $200 \mathrm{kV}$. The two-way actuation strain without external load was measured using a dynamic 
mechanical analyzer (DMA, TA Q800). The actuation strain under different compressive stresses upon cooling was also measured using the DMA. In order to prevent bending of the sample under compressive stress, the copper tube with the inner diameter of $0.5 \mathrm{~mm}$ was added on the outside of the composite wire during the tests. The in-house heat bilges cold shrink of the DMA through the cooling-heating cycle was measured using a quartz sheet with ultra-small thermal expansion coefficient $\left(5.3^{*} 10^{-7}\right)$. The actuation strain data shown in our paper has eliminated the effect of the heat bilges cold shrink of the DMA. The actuation stress output on heating of the sample was measured under position constraint by an Instron testing machine with a temperature-controlled box.

In-situ synchrotron X-ray diffraction measurements were performed at the 11-ID-C beamline of the Advanced Photon Source at Argonne National Laboratory. High energy $\mathrm{X}$-rays of $115 \mathrm{keV}$ energy and $0.6 \mathrm{~mm} \times 0.6 \mathrm{~mm}$ beam size were used to obtain 2D diffraction patterns in the transmission geometry using a Perkin-Elmer large area detector placed at $1.8 \mathrm{~m}$ from the sample. The 2D diffraction patterns were collected during tensile deformation using a home-made mechanical testing device. Gaussian fits were used to determine diffraction peak positions. Error of the $d$-spacing strain measurement is estimated to be smaller than $0.1 \%$.

\section{EXPERIMENT RESULTS}

Figure 1 shows the typical microstructure of the $\mathrm{Nb}$ nanoribbon-nanocrystalline $\mathrm{NiTi}$ composite wire after the prior thermo-mechanical treatments (a tensile deformation of $12 \%$ and a $20 \rightarrow 200 \rightarrow 20^{\circ} \mathrm{C}$ heating-cooling cycle). Figure 1a is a TEM bright-field micrograph of a longitudinal view of the composite wire and Figure 1b is a STEM micrograph of a cross section of the composite wire. It is seen that the composite contained $\mathrm{Nb}$ nanoribbones of 10-30 $\mathrm{nm}$ in thickness and 50-200 $\mathrm{nm}$ in width. The Nb nanoribbons are all well dispersed and aligned along the wire axial direction in the nanocrystalline NiTi matrix with grain sizes in the range of $20-50 \mathrm{~nm}$. The volume fraction of the $\mathrm{Nb}$ nanoribbones is about $12 \%$, as estimated from TEM observation. Figure 1c shows a high-resolution TEM image of the Nb-nanoribbon/NiTi-matrix interface, revealing a clean and well-joined interface. Figure 1d shows a 2D high-energy X-ray diffraction (HE-XRD) pattern of the composite wire, as seen from a lateral direction to the wire length. The composite wire is seen to contain BCC-Nb and B2-NiTi phases, and the $\mathrm{Nb}$ nanoribbons have a strong [110] orientation parallel to the wire axial direction. 

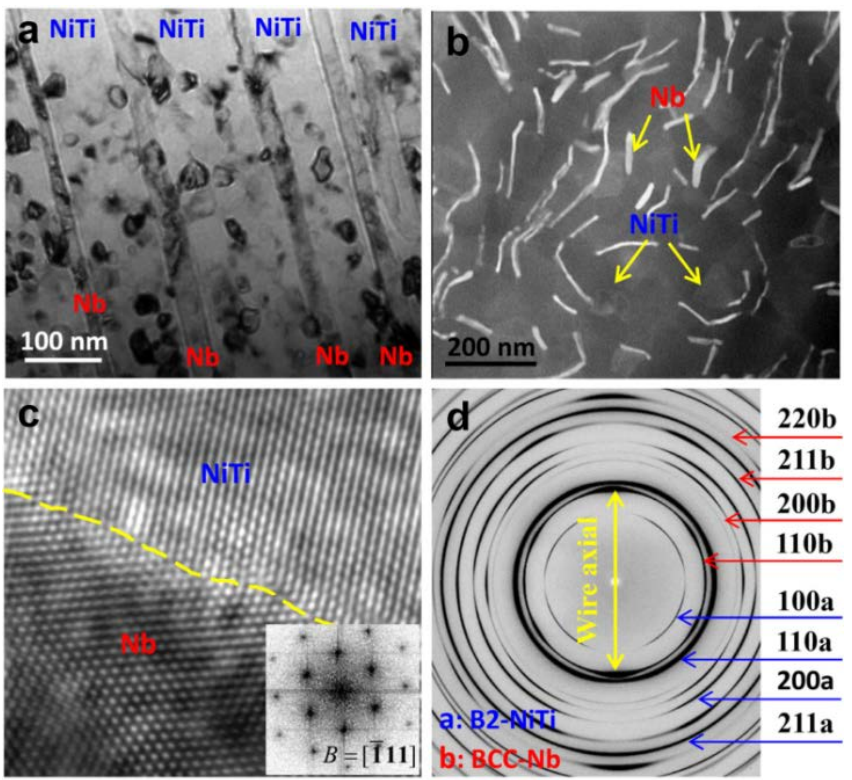

Figure 1. Typical microstructure of the $\mathrm{Nb}$ nanoribbon-nanocrystalline NiTi composite wire. (a) TEM bright-field micrograph of a longitudinal section. (b) STEM image of a cross section (bright regions, cross sections of nanoribbons; dark regions, NiTi matrix). (c) High-resolution TEM image of the Nb-nanoribbon/NiTi-matrix interface. (d) 2D HE-XRD pattern seen from a lateral direction to the wire length.

Figure 2 shows the two-way actuated properties of the composite wire. The sample has been pre-deformed to $12 \%$ tensile strain at room temperature and then gone through a $20 \rightarrow 200 \rightarrow 20{ }^{\circ} \mathrm{C}$ heating-cooling cycle to establish the internal residual stress coupling between $\mathrm{Nb}$ nanoribbons and NiTi matrix (Figure S2). Figure 2a shows the output strain-temperature curves of the sample without external load. It is seen that the sample exhibited an output strain of 3.2\% in elongation upon cooling and in shorten upon heating, and excellent cyclic stability. The actuation stress output upon heating of the composite wire was measured under position constraint using a tensile testing machine. Figure $2 \mathrm{~b}$ shows the actuation stress-temperature curve of the composite upon heating, demonstrating a maximum actuation stress output of $934 \mathrm{MPa}$, which is about twice higher than that of the reported $\mathrm{NiTi}$ SMAs. ${ }^{19}$
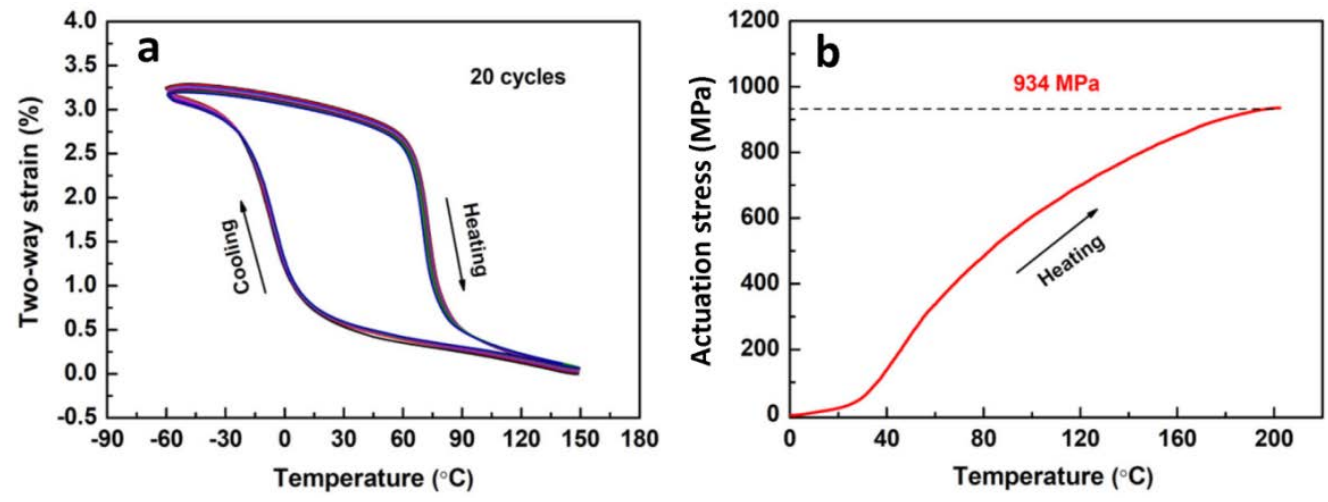

Figure 2. Two-way actuated properties of the composite wire. (a) Output strain-temperature curve of the composite without external load. (b) Actuation stress-temperature curve of the 
composite measured under position constraint upon heating.

To measure the actuation stress output of the composite upon cooling, different magnitude of compressive stresses were applied on the composite along wire axial direction during cooling. Figure 3a shows the output strain-temperature curves of the composite under different constant compressive stresses upon cooling. It is seen that the maximum output strain gradually decreases with increasing applied compressive stress, with $1.35 \%$ output strain at compressive stress of $80 \mathrm{MPa}$. Figure 3b shows the effect of applied compressive stress on the maximum strain output and the mechanical work output. It is seen that simple extrapolation of the curve to nil strain suggests that the maximum compressive stress about the composite wire is able to overcome is $134 \mathrm{MPa}$, in other words, the maximum actuation stress upon cooling of the composite wire under position constraint is $134 \mathrm{MPa}$, which is significantly higher than that (5 50 MPa) of the reported two-way memory SMAs. ${ }^{15-16}$ It is also noted that the maximum mechanical work output on cooling is $1.08 * 10^{6} \mathrm{~J} / \mathrm{m}^{3}$ at a resistive stress (compression) of $80 \mathrm{MPa}$, which is about 5 times more than that $\left(2.0^{*} 10^{5} \mathrm{~J} / \mathrm{m}^{3}\right)$ of the reported two-way memory SMAs. ${ }^{15}$
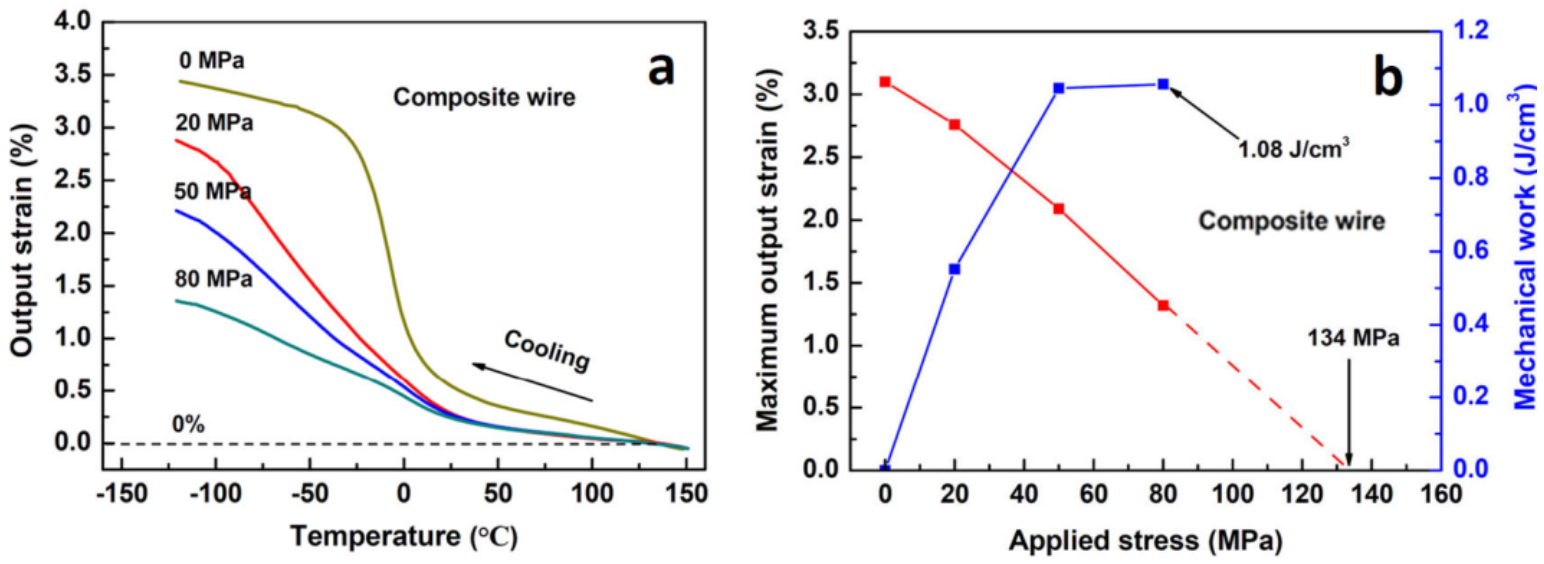

Figure 3. Two-way actuated properties of the composite wire upon cooling. (a) Output strain-temperature curves under different constant compressive stresses. (b) Effect of applied compressive stress on the maximum strain output and the mechanical work output.

To uncover the underlying mechanism of the two-way actuation of the composite wire, in-situ HE-XRD experiment was carried out on the composite through a tensile deformation of $12 \%$ at room temperature. Figure S3 shows a HE-XRD pattern of the sample before the deformation, indicating that it contained BCC-Nb and B19'-NiTi phases. Figure 4a shows the tensile stress-strain curve of the composite, and Figure 4b shows the corresponding in-situ HE-XRD patterns. Evolution of $\mathrm{Nb}$ (110) and B19'-NiTi (001) $d$-spacing strains with respect to applied tensile strain in the wire axial direction are shown in Figure $4 \mathrm{c}$ and $4 \mathrm{~d}$, respectively. It is seen that the $\mathrm{Nb}$ nanoribbons exhibited a plastic yield at $4.7 \%$ lattice strain (point $\mathrm{A}$ in Figure 4c) and a maximum lattice strain of 5.4\% (point B in Figure 4c) upon loading, and a residual tensile lattice strain of 2.2\% after unloading (point $C$ in Figure $4 \mathrm{c}$ ). The occurrence the residual lattice strain can be understood as following. Upon loading to $12 \%$ global strain, the martensitic NiTi matrix underwent an elastic deformation of self-accommodated 
martensite to $\sim 1 \%$ global strain (O-A in Figure $4 \mathrm{~d}$ ), followed by martensite variant reorientation and an elastic deformation of the oriented variants to $\sim 12 \%$ global strain (A-C in Figure 4d). At the same time, the Nb nanoribbons underwent an elastic deformation up to $\sim 6 \%$ global strain (O-A in Figure 4c) followed by a plastic deformation (A-B in Figure 4c) to the end of the deformation cycle at $12 \%$ global strain. Upon unloading, the NiTi matrix and the $\mathrm{Nb}$ nanoribbons both underwent elastic strain recovery. However, the elastic strain of the NiTi matrix (2.2\% in Figure $4 d$ ) is smaller than that of the Nb nanoribbons (5.4\% in Figure 4c), thus the NiTi matrix hinders the full elastic recovery of the Nb nanoribbons upon unloading, causing tensile strain in the $\mathrm{Nb}$ nanoribbons (2.5\% in Figure 4c) and compressive strain in the NiTi matrix ( $0.36 \%$ in Figure $4 d)$ after unloading. To better understand the above deformation mechanisms, the schematics of the deformation processes of the $\mathrm{Nb}$ nanoribbons and the NiTi matrix through the tensile deformation of 12\% are shown in Figure S4.
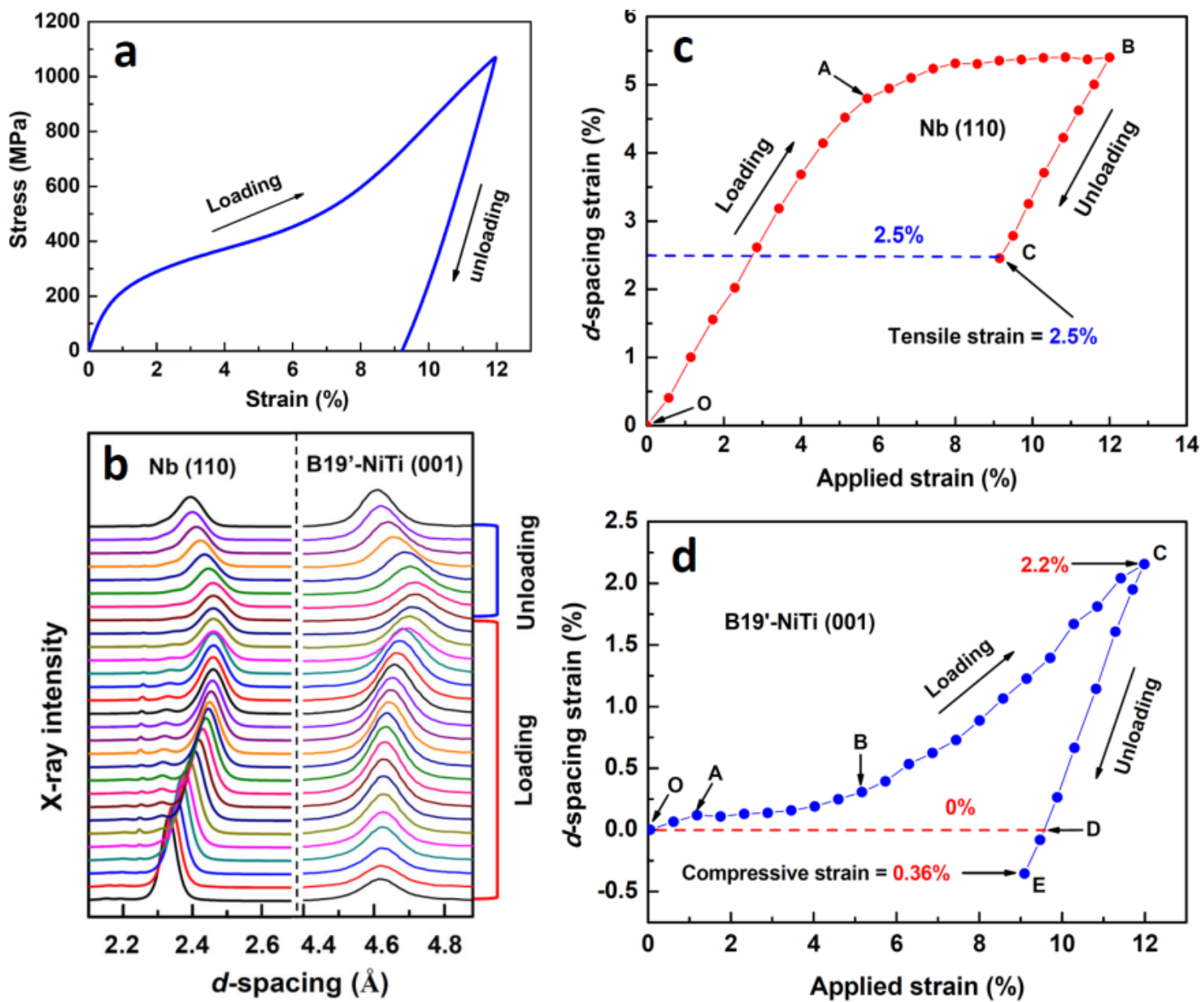

Figure 4. In-situ HE-XRD analysis of the composite wire through a $12 \%$ tensile deformation. (a) The tensile stress-strain curve. (b) HE-XRD patterns collected at different strain levels during the deformation cycle. (c) Evolution of $\mathrm{Nb}$ (110) $d$-spacing strain in the wire axial direction as a function of the applied tensile strain. (d) Evolution of B19'-NiTi (001) $d$-spacing strain in the wire axial direction as a function of the applied tensile strain.

The sample after the prior tensile deformation was subjected to a $20 \rightarrow 200 \rightarrow 20{ }^{\circ} \mathrm{C}$ heating-cooling cycle to induce shape recovery of the NiTi matrix. Figure 5a shows the 
strain-temperature curve of the sample through the thermal cycle. During this thermal cycle, in-situ HE-XRD was also measured, and the diffraction patterns are shown in Figure 5b. Figure 5c shows the evolution of the $d$-spacing strain for the Nb (110) planes perpendicular to the wire axial direction during the thermal cycle. It is seen that the $\mathrm{Nb}$ nanoribbons had a $2.5 \%$ tensile strain at the start of the thermal cycle (inherited from the prior deformation) and underwent a contraction upon heating to above $160{ }^{\circ} \mathrm{C}$ (apparently associated with the reverse transformation of the NiTi matrix) to a compressive elastic strain of $2.1 \%$. Subsequent cooling to $20{ }^{\circ} \mathrm{C}$ did not cause much change to the strain state of the $\mathrm{Nb}$ nanoribbons.
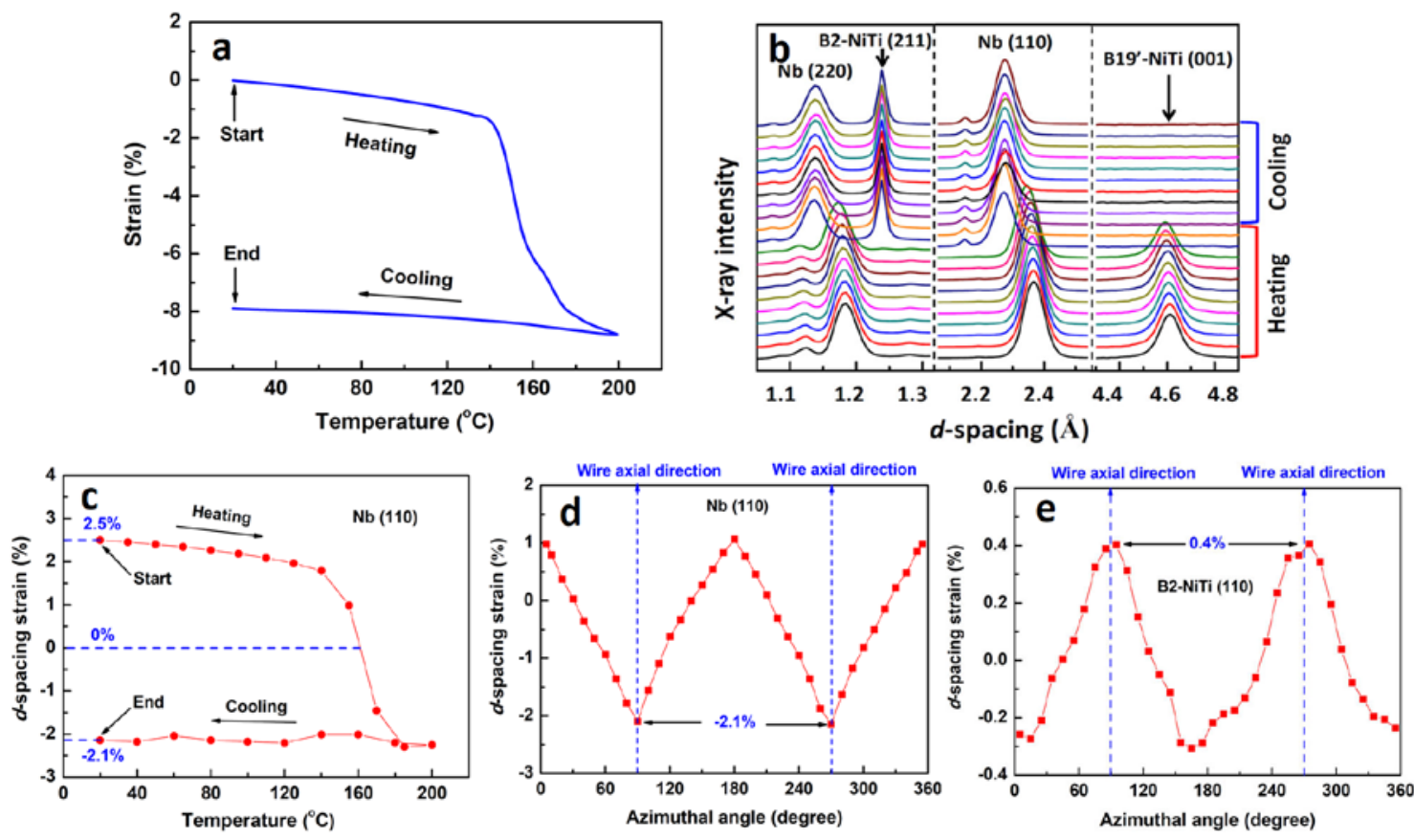

Figure 5. In-situ HE-XRD analysis of the composite wire through the heating-cooling cycle. (a) The output strain-temperature curve. (b) In-situ HE-XRD patterns. (c) Evolution of $d$-spacing strain with respect to temperature for the $\mathrm{Nb}$ (110) planes perpendicular to the wire axial direction. (d-e) Plots of $d$-spacing strain for Nb (110) and B2-NiTi (110) planes versus azimuthal angle of the 2D HE-XRD pattern after the thermal cycle.

Using the full 2D HE-XRD pattern of the sample after the thermal cycle, as shown in Figure $1 \mathrm{~d}$, the $\mathrm{Nb}(110)$ and B2-NiTi (110) $d$-spacing strains in different directions of the wire can be determined. Figures $5 d$ and 5e show the $\mathrm{Nb}$ (110) and B2-NiTi (110) $d$-spacing strains as functions of the azimuthal angle $(\eta)$ measured from the lateral direction of the composite wire, respectively. It is seen that the lattice strains of the Nb nanoribbons and the NiTi matrix have a $180^{\circ}$ symmetry. The $\mathrm{Nb}$ nanoribbons had a compressive elastic strain of $2.1 \%$ in the wire axial direction and a $1.0 \%$ tensile strain in the lateral direction after the thermal cycle. Correspondingly, the NiTi matrix had a tensile elastic strain of $0.4 \%$ along the wire axial direction and a $0.3 \%$ compressive strain in the lateral direction. The tensile strain in the $\mathrm{Nb}$ nanoribbons and the compressive strain in the NiTi matrix along the wire axial direction are opposite to the lattice strains of the two components after the deformation cycle 
(Figures 4c and 4d). This can be understood as following. Upon heating, the NiTi matrix underwent a contraction of $8 \%$ (Figure $5 \mathrm{a}$ ) via the reverse martensitic transformation (B19' $\rightarrow$ B2) (Figure 5b). The Nb nanoribbons embedded in the NiTi matrix, on the other hand, had $2.5 \%$ elastic tensile strain stored prior to the heating (Figure $4 \mathrm{c}$ ), thus the $\mathrm{Nb}$ nanoribbons hinder the full shape recovery of the NiTi matrix upon heating, causing compressive strain in the $\mathrm{Nb}$ nanoribbons and tensile strain in the NiTi matrix. This result also explains clearly that after the thermal cycle, the tensile elastic strain (thus stress) in the NiTi matrix along the wire axial direction, as induced by the $\mathrm{Nb}$ nanoribbons, provides the guide for the two-way actuation in subsequent cycles.

In-situ HE-XRD experiment was also conducted on the composite wire through a two-way actuation cycle (Figure 2a), and the diffraction patterns are shown in Figure 6a. It is seen that the intensity of the B2-NiTi diffraction peaks decreases gradually and the intensity of the B19'-NiTi diffraction peaks increases with decreasing temperature on cooling, implying the $\mathrm{B} 2 \rightarrow \mathrm{B} 19$ ' martensitic transformation. Upon heating, the intensities of both B2-NiTi and B19'-NiTi diffraction peaks show the opposite trends, indicating the B19' $\rightarrow$ B2 reverses martensitic transformation. Figure $6 \mathrm{~b}$ shows the evolution of the $d$-spacing strain for the $\mathrm{Nb}$ (110) planes perpendicular to the wire axial direction through the two-way actuation cycle. It is seen that the $\mathrm{Nb}$ nanoribbons had a $2.1 \%$ compressive elastic strain at the start of tow-way actuation cycle (inherited from the prior thermal cycle). The compressive elastic strain was released upon cooling and regained upon heating.
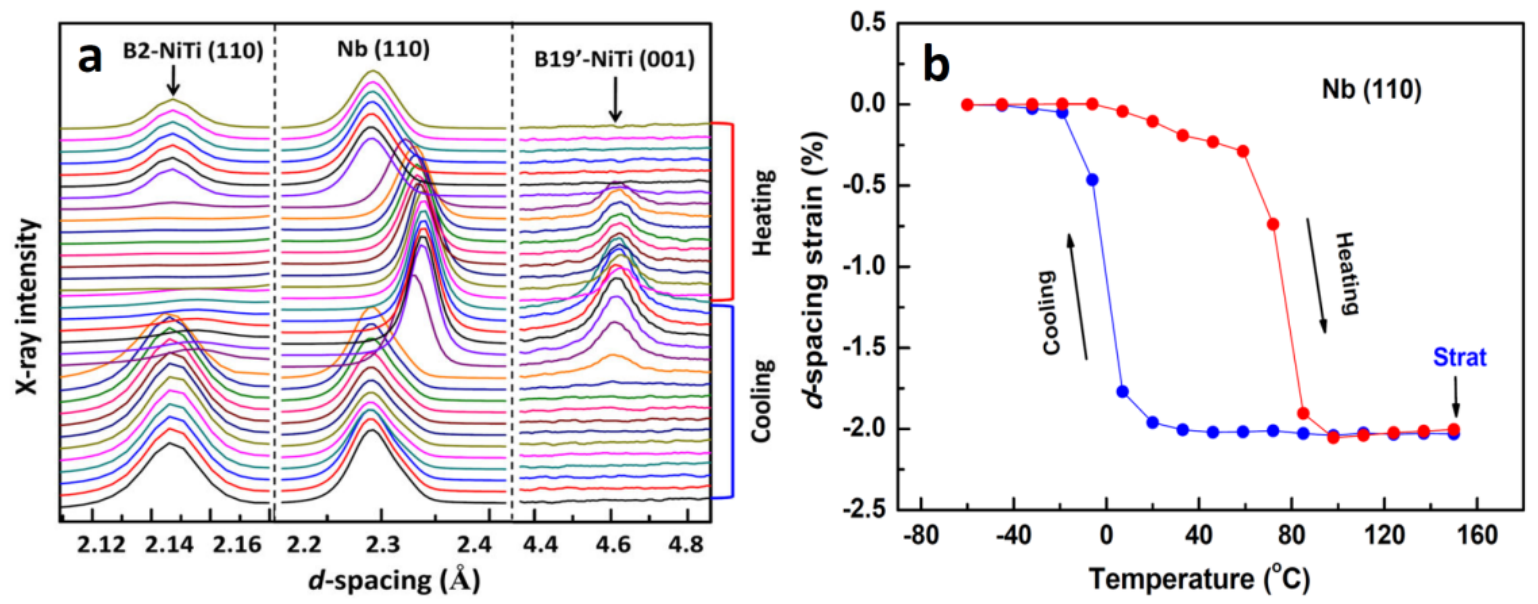

Figure 6. In-situ HE-XRD analysis of the composite wire through the two-way actuation cycle. (a) In-situ HE-XRD patterns. (b) Evolution of $d$-spacing strain with respect to temperature for the $\mathrm{Nb}(110)$ plane perpendicular to the wire axial direction.

\section{DISCUSSION}

It is known that a two-way memory effect can be developed in NiTi SMAs through certain thermo-mechanical treatment, such as shape memory training, ${ }^{20-21}$ pseudoelastic training, ${ }^{22}$ and thermal cycling training under a constant stress. ${ }^{23}$ It is commonly understood that the essence of these different treatments is to create directional internal stress fields, which may direct the nucleation and growth of preferentially orientated martensite variants in subsequent thermal cycles without the influence of an applied stress, thus realizing the two-way memory effect. $^{10,20-24}$ Prior deformation slightly beyond the strain limit of the martensitic 
transformation as a means to develop two-way memory effect is to create such an anisotropic dislocation structure in NiTi SMAs. ${ }^{10,22,24}$

The two-way actuation of the Nb nanoribbon-nanocrystalline NiTi composite studied in this work is induced by a new mechanism, via a long range directional stress coupling between the $\mathrm{Nb}$ nanoribbons and the NiTi matrix, as opposed to the anisotropic short range stress fields associated with the deformation-induced dislocations. The presence of the long range coupling stresses (elastic lattice strains) between the $\mathrm{Nb}$ nanoribbons and the $\mathrm{NiTi}$ matrix is evident in Figures 5d and 5e. The mechanism for the generation of such internal coupling stresses (strains) through the prior thermo-mechanical treatments is schematically illustrated in Figure S5. Figure S6-a shows the 2D HE-XRD pattern of the composite at the cooling end of the two-way actuation cycle, and Figure S6-b shows the variation of X-ray diffraction intensity of B19'-NiTi <001> planes versus the azimuth angle along the Debye-Scherrer ring indicated in Figure S6-a. It is seen that the diffraction intensity of B19'-NiTi (001) planes along the Debye-Scherrer ring concentrates approximately equally at four locations, separated by $\sim 60^{\circ}$, indicating that the NiTi matrix at the cooling end of the two-way actuation cycle was composed of spatially preferred martensitic variants. The result confirms the hypothesis that the directional internal stresses in the NiTi matrix caused by the $\mathrm{Nb}$ nanoribbons guide the formation of selected preferential variants upon cooling for the two-way actuation observed. The mechanism for the internal coupling stress between $\mathrm{Nb}$ nanoribbons and NiTi matrix inducing the two-way actuation of the composite wire is schematically illustrated in Figure S7.

To further verify the understanding that the two-way actuation obtained in this composite is due to the coupling stresses between the Nb nanoribbons and the NiTi matrix, and not associated with deformation induced dislocations, we fabricated a Ti-50.at\% Ni reference sample for comparison. The Ti-50.at\% Ni sample was produced by severe cold wire-drawing followed by a $400{ }^{\circ} \mathrm{C}$ annealing. Such treatment produced a practically identical microstructure with nanograins of the order of $20-50 \mathrm{~nm}$ in size to that of the NiTi matrix of the composite wire. The TiNi wire was then subjected to the same thermo-mechanical treatment to that of the composite wire (a tensile deformation of $12 \%$ followed by a $20 \rightarrow 200 \rightarrow 20{ }^{\circ} \mathrm{C}$ heating-cooling cycle). After such treatments, the NiTi wire exhibited a two-way strain output of $\sim 0.5 \%$ (Figure S8), which is much smaller than that ( $3.2 \%)$ of the composite wire. This implies that the two-way actuation of the composite is indeed induced by the coupling stresses between the Nb nanoribbons and the nanocrystalline NiTi matrix. This understanding is also consistent with the expectation that nanograins are incapable of generating internal dislocations during mild deformation.

It is commonly considered that the driving stress upon cooling of the two-way memory SMAs is determined by the critical stress for martensite reorientation. ${ }^{10,15-16}$ Because the critical stress for martensite reorientation of the SMAs is usually only a few tens of MPa, ${ }^{10-11}$ a low resistive stress can change the orientation of martensite variants, thus resulting in the low stress output of the SMAs upon cooling. For the composite wire, the Nb nanoribbons were subjected to a compressive elastic strain of $2.1 \%$ after the prior thermo-mechanical treatment, as shown in Figure 5c and 5d. The large compressive elastic strain was gradually released upon cooling and regained upon heating in the two-way actuation process of the composite wire, as shown in Figure 6b. It is evident that the massive numbers of $\mathrm{Nb}$ 
nanoribbons in compressive state serve as built-in nano bias springs, providing high stress output and high work output upon cooling for the composite wire.

It is known that the maximum actuation stress on heating of the two-way memory SMAs is determined by the yield strength (the critical stress for dislocation slip) of the SMAs. ${ }^{10,19}$ For the composite wire, the grain size of the NiTi matrix is in nanometer scale $(20-50 \mathrm{~nm})$, expecting that the NiTi matrix has a high yield strength, thus it is considered that the high yield strength of the NiTi matrix results in the high actuation stress on heating of the composite wire. To further verify this understanding, we obtained the composite wires where the NiTi matrix has different yield strength (different grain sizes) by changing the prior annealing temperature. It is evident that the maximum driving stress of the composite wire gradually decreases with decreasing yield strength (increasing grain size) of the NiTi matrix from Figure S9. This result is consistent with our understanding that the high yield strength of the NiTi matrix results in the high actuation stress on heating of the composite wire.

\section{CONCLUSIONS}

A novel two-way actuation nanocomposite wire was fabricated by severe cold wire-drawing of a Nb-NiTi hypo-pseudoeutectic casting and thermo-mechanical treatments. The composite simultaneously provides high actuation stresses and large actuation strains during both heating and cooling processes. It is revealed that the massive number of $\mathrm{Nb}$ nanoribbons serving as built-in nano bias springs provides the high actuation stress and high work output upon cooling and the SMA matrix with high yield strength offers the high actuation stress upon heating. The two-way actuation mechanism of the composite is entirely different from that the reported two-way memory SMAs. Moreover, the two-way actuation composite wire with small volume and simple construct is in favour of the miniaturization and simplification of actuators.

\section{- AUTHOR INFORMATION \\ Corresponding Author \\ *E-email: haoshijie@cup.edu.cn}

\section{Notes}

The authors declare no competing financial interest

\section{- ACKNOWLEDGEMENTS}

This work was supported by the Key Program of NSFC (51231008), the NSFC (51471187, 11474362 and 51571211), Beijing Natural Science Foundation (2152026), the ARC grant (DP140103805), the Fok Ying-Tong Education Foundation for Young Teachers in the Higher Education Institutions of China (151045), State Key Lab of Advanced Metals and Materials (2014-ZD01), and Science Foundation of China University of Petroleum-Beijing (2462013YJRC005). The use of the Advanced Photon Source was supported by the US Department of Energy, Office of Science, and Office of Basic Energy Science under Contract No. DE-AC02-06CH11357.

\section{- REFERENCES}


(1) Kim, B.; Lee, M. G.; Lee, Y. P.; Kim, Y. I.; Lee, G. H. An earthworm-like micro robot using shape memory alloy actuator. Sens. Actuators, A. 2006, 125, 429-437.

(2) Villanueva, A.; Smith, C.; Priya, S. A biomimetic robotic jellyfish (Robojelly) actuated by shape memory alloy composite actuators. Bioinsp. Biomim. 2011, 6, 036004.

(3) Humbeeck, J. V. Non-medical applications of shape memory alloys. Mater. Sci. Eng., A. 1999, 273-275, 134-148.

(4) Furst, S. J.; Bunget, G.; Seelecke, S. Design and fabrication of a bat-inspired flapping-flight platform using shape memory alloy muscles and joints. Smart Mater. Struct. 2013, 22, 014011.

(5) EI Feninat, F.; Laroche, G.; Fiset, M.; Mantovani, D. Shape Memory Materials for Biomedical Applications. Adv. Eng. Mater. 2002, 4, 91-104.

(6) Chonan, S.; Jiang, Z. W.; Tani, J.; Orikasa, S.; Tanahashi, Y.; Takagi, T.; Tanaka, M.; Tanikawa, J. Development of an artificial urethral valve using SMA actuators. Smart Mater. Struct. 1997, 6, 410-414.

(7) Laurentis, K. J. D.; Mavroidis, C. Mechanical design of a shape memory alloy actuated prosthetic hand. Technol Health Care. 2002, 10, 91-106.

(8) Uchino K. Piezoelectric actuators and ultrasonic motors. Kluwer Academic Publisher, 1997.

(9) Fukuda, T.; Hosokai, H.; Ohyama, H.; Hashnoto, H.; Arai, F. Giant Magnetostrictive Alloy (GMA) Applications to Micro Mobile Robot as a Micro Actuator without Power Supply Cables. Proc. IEEE Conf. Micro Electro Mechanical Systems, 1991, 210-215.

(10) Otsuka, K.; Wayman, C. M. Eds, Shape memory materials (Cambridge Univ. Press, Cambridge, 1998).

(11) Otsuka, K.; Ren, X. Physical Metallurgy of Ti-Ni-Based Shape Memory Alloys. Prog. Mater. Sci. 2005, 50, 511-678.

(12) Pelrine, R. E.; Kornbluh, R. D.; Joseph, J. P. Electrostriction of polymer dielectrics with compliant electrodes as a means of actuation. Sens. Actuators, A. 1998, 64, 77-85.

(13) Krulevitch, P.; Lee, A. P.; Ramsey, P. B.; Trevino, J. C.; Hamilton, J.; Northrup, M. A. M. A. Thin film shape memory alloy microactuators. J. Microelectromech. Syst. 1996, 5, 270-282.

(14) Miyazaki, S.; Ishida, A. Martensitic transformation and shape memory behavior in sputter-deposited TiNi-base thin films. Mater. Sci. Eng., A. 1999, 273-275, 106-133.

(15) Stalmans, R.; JV Humbeeck, J. V.; Delaey, L. The two way memory effect in copper-based shape memory alloys-thermodynamics and mechanisms. Acta Metall. Mater. 1992, 40, 2921-2931.

(16) Larochette, P. A.; Ahlers. M. Grain-size dependence of the two-way shape memory effect obtained by stabilisation in Cu-Zn-Al crystals. Mater. Sci. Eng., A. 2003, 361, 249-257

(17) Huang. W. On the selection of shape memory alloys for actuators. Mater. Des. 2002, 23, 11-19.

(18) Hao, S. J.; Cui, L. S.; Jiang, D. Q.; Han, X. D.; Ren, Y.; Jiang, J.; Liu, Y. N.; Liu, Z. Y.; Mao, S. C.; Wang, Y. D.; Li, Y.; Ren, X. B.; Ding, X. D.; Wang, S.; Yu, C.; Shi, X. B.; Du, M. S.; Yang, F.; Zheng, Y. J.; Zhang, Z.; Li, X. D.; Brown, D. E.; Li, J. A 
Transforming Metal Nanocomposite with Large Elastic Strain, Low Modulus, and High Strength. Science 2013, 339, 1191-1194.

(19) Yan, X. J.; Ge, Y. L.; Humbeeck, J. V. Influence of Different Thermo-mechanical Cycling Routes on Recovery Stresses of Annealed NiTi Wires. J. Mater. Eng. Perform. 2016, 25, 267-273.

(20) Perkins, J.; Sponholz, R. O. Stress-induced martensitic-transformation cycling and 2-way shape memory training in Cu-Zn-Al alloys. Metall Trans A. 1984, 15, 313-321.

(21) Reyhani, M. M.; McCormick, P. G. Proc of ICOMAT-86. Japan Institute of Metals, 1986, 896.

(22) Contardo, L.; Guenin, G. Training and two way memory effect in Cu-Zn-Al alloy. Acta Metall. Mater. 1990, 38, 1267-1272.

(23) Liu, Y. and McCormick, P. G. Factors influencing the development of two-way shape memory in NiTi. Acta Metall. Mater. 1990, 38, 1321-1326.

(24) Wada, K.; Liu, Y. On the two-way shape memory behavior in NiTi alloy-An experimental analysis. Acta Mate. 2008, 56, 3266-3277. 